\title{
Continuous wave Doppler ultrasonography in the detection of carotid stenosis and occlusion
}

\author{
PRD HUMPHREY, PG BRADBURY \\ From the National Hospital for Nervous Diseases, Queen Square, London, UK
}

SUMMARY Directional continuous wave Doppler ultrasonography has been used to assess the extracranial carotid arteries of over 500 patients, 90 of whom have had angiography performed. The angiographic and Doppler findings are compared, showing that this simple ultrasound technique is able to detect carotid stenosis and occlusion, but is unreliable in the detection of mild atheromatous change. In this series, the method detected $90 \%$ of the angiographically proven carotid stenoses, being considerably more accurate than routine auscultation. It would seem that continuous wave ultrasonography is a safe and practical screening test in those patients in whom arteriography is being considered.

About $20 \%-30 \%$ of patients with carotid transient ischaemic attacks (TIAs) referred to a neurological centre are found to have a surgically correctable lesion;' the majority of the patients thus undergo unnecessary and potentially hazardous angiography. This is one of the factors responsible for the marked variation in the percentage of patients undergoing angiography for the investigation of TIAs in different centres throughout the United Kingdom ( 3 to $100 \%){ }^{2}$

Prior to the introduction of digital vascular imaging, ultrasound formed the basis of most of the techniques used for the non-invasive investigation of the carotid arteries as a preliminary to angiography. Over the past two years continuous wave Doppler ultrasonography has been used at this hospital to assess such patients. The results have been compared with the findings of conventional angiography, and with the presence of a carotid bruit, which is the most reliable clinical guide to carotid stenosis. ${ }^{34}$

\footnotetext{
Address for reprint requests: P R D Humphrey, Regional Department of Medical \& Surgical Neurology, Walton Hospital, Rice Lane, Liverpool, L9 1AE, UK.
}

Received 4 January 1984 and in revised form 29 March 1984. Accepted 1 April 1984

\section{Methods}

A continuous wave directional Doppler flowmeter using transducers with non-focused beams of 4 and $8 \mathrm{MHz}$ (System Sonotechnik model LC2) was used to assess the flow profiles in the supraorbital and supratrochlear arteries around the eye, and the flow in the extracranial carotid arteries in the neck, in order to screen for haemodynamically significant stenoses and occlusions in these vessels. ${ }^{5-7}$

In continuous wave ultrasonography, high frequency sound waves are reflected by moving red blood cells causing an associated Doppler shift; the degree of this shift is proportional to the velocity of the blood. In the presence of a stenosis, the velocity of red blood cells through the narrowed segment rises, producing an increase in the Doppler shift. This may be the only abnormality in a mild stenosis. Tight stenotic lesions situated in the internal carotid artery can also produce a reduction in the flow in the ipsilateral common carotid artery detectable with this technique. The supraorbital and supratrochlear arteries constitute part of the watershed circulation between the internal and external carotid arteries; the direction of the flow in these orbital vessels is dependant on the peripheral resistance in the carotid arteries. As the pressure in the ophthalmic artery is normally greater than that in the terminal branches of the external carotid artery, the normal flow in the supratrochlear and supraorbital arteries is out of the orbit. However, if a haemodynamically significant stenosis is present in the internal carotid artery, the perfusion pressure to the ophthalmic artery may be progressively reduced and may eventually fall below the perfusion pressure in the branches of the external carotid artery, thereby reversing 
flow in the supraorbital and supratrochlear arteries. The detection of such altered perfusion is greatly facilitated by digital compression of the superficial temporal and facial arteries, which by temporarily reducing the pressure from the external carotid artery may unmask low internal or dominant external carotid flows. This study combined analysis of the supraorbital, supratrochlear and the common, internal and external carotid artery signals, as described above, to detect haemodynamically significant lesions at various sites in the extracranial carotid arteries.

Over the past two years Doppler studies have been performed on over 500 patients at this hospital. Of these, 90 came to angiography, most having conventional catheter arteriograms but six having digital vascular imaging. The angiograms were reviewed blind by a consultant neuroradiologist, unaware of the Doppler findings. The internal carotid artery was classified as normal, occluded, stenosed (31-99\% narrowing) or atheromatous (up to $30 \%$ narrowing); these results were compared with the Doppler findings, as was the presence of a carotid artery bruit on clinical examination.

\section{Results}

\section{Correlation with angiography}

Angiographic results were available for 154 carotid arteries. Twenty-five vessels with arteriographically proven carotid occlusion were correctly diagnosed by Doppler study (table). The one false positive result, in which no signal was detected from the internal carotid artery suggesting occlusion, was found on angiography to be due to a normal extracranial segment to the internal carotid artery with a tight intracranial stenosis producing a marked reduction in flow in the proximal segment.

Of the 39 vessels with angiographically proven internal carotid artery stenosis, 35 were correctly diagnosed by Doppler study. There were four false negatives in this group in which arteriography showed stenotic lesions. In all four cases, turbulence suggestive of atheroma was noted but no high frequency stenotic signal detected. In the one false positive case in this group a high frequency stenotic signal was detected, but angiography showed severe atheroma but no stenosis.

Table The Doppler and angiographic findings of the internal carotid artery in 154 carotid arteries (90 patients)

\begin{tabular}{clc}
\hline Carotid occlusions & \multicolumn{2}{l}{ Angiography } \\
& + & - \\
DOPPLER + & 25 & 1 \\
- & 0 & \\
Carotid stenosis (31-99\% narrowing) & Angiography & \\
& + & - \\
DOPPLER + & 35 & 1 \\
- & 4 & \\
Carotid atheroma (up to 30\% & & Angiography \\
narrowing) & + & - \\
DOPPLER + & 15 & 9 \\
- & 13 & \\
\hline
\end{tabular}

In those patients with angiographically proven mild atheroma, the Doppler and angiographic findings agreed for 15 vessels. In the nine false positive results, turbulence suggestive of atheroma was present on Doppler examination, but angiography showed either minimal atheroma or a normal vessel. In the 13 false negative cases, the Doppler result was normal, but angiography showed clear atheroma. However, in no case was a significant stenosis found. In 51 arteries both the Doppler and angiographic findings were normal.

\section{Correlation with bruits}

Of the 39 arteries with carotid stenoses on angiography, a carotid bruit was only present in 27 . In the 12 "bruit negative" examples, three had severe stenoses (greater than $90 \%$ ), six had moderate stenoses (65-90\%) and three had mild stenoses (31$50 \%)$. The Doppler study correctly detected nine of these 12 "bruit negative" stenoses.

There was 13 false positive results, of which two had normal angiograms, four showed irregularity of the internal carotid only, three had an external carotid stenosis and four had internal carotid occlusions (two being on the same side as the bruits and two on the opposite side of the bruit). Doppler study correctly assessed 12 of these 13 false positive bruits. The one incorrectly assessed bruit, thought on Doppler study to represent an internal carotid artery stenosis, proved on angiography to be an external carotid artery stenosis.

\section{Discussion}

The continuous wave Doppler technique used in this study has been shown to be effective in detecting carotid stenoses and occlusions, confirming the findings of Hennerici et al. ${ }^{8}$ In our study all 25 patients with carotid occlusions were successfully detected with one false positive result. Of the 39 patients with internal carotid artery stenoses near the bifurcation, $90 \%$ were correctly detected. However, the technique described herein is of limited use in the detection of mild atheromatous disease. The more refined ultrasonic methods combining Doppler flow and echo imaging techniques enable the visualization of mild degrees of atheroma in addition to giving information about flow. ${ }^{10}$ However, these techniques are considerably more expensive and for many situations a simple Doppler flowmeter is all that is required to give useful information with regard to possible carotid occlusion and stenosis.

Carotid angiography remains the standard technique for the investigation of the carotid arteries in patients with TIAs. However, the techniques still have a small but significant morbidity, usually esti- 
mated at about a $1 \%$ risk of stroke or death. However, a recent prospective study in which each patient was assessed by a neurologist suggests that the risk may be as high as 5\%." Digital vascular imaing may lessen these risks but, unfortunately, the technique does not produce adequate views of the carotid bifurcation in all patients. ${ }^{12}$

Because of the risks, many clinicians will only submit patients to angiography if it is felt that a stenosis is likely to be present on the basis of clinical history and examination. A localised bruit is the best clinical guide. This study, in addition to others, ${ }^{8}$ has shown that the presence of a bruit is of limited value. Whilst a bruit was present in $27(69 \%$ of our patients with internal carotid stenosis), 12 (31\%) patients were without a bruit. The Doppler examination correctly detected nine of these 12 false negatives. Furthermore, there were 13 false positive bruits, of which 12 were correctly assessed by this technique. Thus of the 25 false negative and false positive bruits in this group the Doppler was correct in 21 cases. The technique was, therefore, clearly superior to auscultation of the neck. The method, and in particular the handling of the transducers, is very much operator sensitive. However, with suitable supervision the authors feel that it is possible to become reasonably competent after approximately 100 studies. Ultrasonic methods have been shown to be increasingly accurate in the assessment of extracranial carotid artery disease. In our opinion it proves a safe, simple and cheap screening test helping clinicians to decide when angiography would be likely to reveal carotid stenoses and occlusions.

We are deeply indebted to Dr Michael Hennerici who was instrumental in helping us to master this technique over his 3 months stay at The National Hospital. We thank Dr John Stevens for assessing the carotid angiograms and Professors $\mathrm{J}$ Marshall and WI McDonald for their help and encouragement with this project.

\section{References}

' Humphrey PRD, Marshall J. Transient ischaemic attacks and strokes with recovery. Stroke 1981;12:765-9.

${ }^{2}$ UK-TIA Study Group. Variation in the use of angiography and carotid endarterectomy by neurologists in the UK-TIA Aspirin trial. Br Med J 1983;1:514-7.

${ }^{3}$ Harrison MJG, Marshall J. Indications for angiography and surgery in carotid artery disease. $\mathrm{Br}$ Med $\mathrm{J}$ 1975; 1:616-7.

${ }^{4}$ Wilson LA, Ross Russell RW. Amaurosis fugax and carotid artery disease: indications for angiography. $\mathrm{Br}$ Med J 1977;2:435-7.

${ }^{5}$ Keller H, Meier W, Yonekawa Y, Kump D. Noninvasive angiography for the diagnosis of carotid artery disease using Doppler ultrasound (carotid artery Doppler). Stroke 1976;7:354-63.

${ }^{6}$ Budingen HJ, Von Reutern GH, Freund HJ. Diagnosis of cerebrovascular lesions by ultrasonic methods. Int J Neurology 1977;11:206-18.

7 Pourcelot L. Diagnostic ultrasound for cerebral vascular diseases. In: Donald I, Levi S, eds. Present and Future of Diagnostic Ultrasound. New York: J Wiley, 1976:141-7.

${ }^{8}$ Hennerici M, Aulich A, Sandmann W, Freund HJ. Incidence of asymptomatic extracranial arterial disease. Stroke 1981;12:750-8.

${ }^{9}$ Sumner DS. Non-invasive investigation of the arterial supply to the brain and eye. In: Charles Warlow, Peter J Morris, eds. Transient Ischaemic Attacks. New York: Marcel Dekker Inc, 1982:177-219.

${ }^{10}$ Woodcock JP. Special ultrasonic method for the assessment and imaging of systemic arterial disease. $\mathrm{Br} J$ Anaesthesia 1981;53:719-30.

"Steiner TJ, McIvor J, Perkin GD, Greenhalgh RM, Clif ford Rose F. In: Greenhalgh RM, Clifford Rose Fọ eds. Progress in Stroke Research 2. London: Pitman? 1983:136-53.

${ }^{12}$ Little JR, Furlan AJ, Modic MT, Weinstein MA. Digital subtraction angiography in cerebrovascular disease. Stroke 1982;13:557-66. 